\title{
Effects of a Distribution of Volume Relaxation Times in the Annealing of BSC Glass
}

\author{
P. B. Macedo and A. Napolitano \\ Institute for Materials Research, National Bureau of Standards, Washington, D.C. 20234
}

(February 20, 1967)

\begin{abstract}
The influence of a distribution of relaxation times is studied in annealing experiments. A tworelaxation time model is proposed, which permits the calculation of the distribution of relaxation times from the crossover data of Spinner and Napolitano as well as that of Ritland. This model also characterizes the structure of any glass in terms of two parameters. Thus, quenched equilibrium as well as nonequilibrium glass were compared with rate cooled glasses with respect to their behavior upon further annealing as well as their conductivity at low temperatures with excellent agreement.

Borosilicate crown glass was found to have a narrow distribution of relaxation times which is particular to associated liquid monomers rather than polymers. The results can be explained in terms of a topological model for the distribution of relaxation times. The temperature dependence of the viscosity is due to a true activation energy rather than a free volume effect.
\end{abstract}

Key Words: Annealing, borosilicate crown glass, glass, index of refraction, relaxation times, two relaxation time model, volume relaxation.

\section{Introduction}

This study deals with the analysis of some annealing experiments performed on a borosilicate glass by Spinner and Napolitano [1]. ${ }^{1}$ They established timeindex of refraction isotherms for their glass in the transformation region. It is well known that the index of refraction of glass at room temperature is dependent on its thermal history, i.e., the type of heat treatment that the parent liquid received as it was cooled through the transformation region. The index of refraction $(N)$ has been used throughout this study because, of the various physical properties which reflect this change in structure, the refractive index of glass can be most accurately measured. It is assumed throughout that the mechanical deformations associated with change of temperature are purely dilatations.

The thermal history of the glass in the transformation region is shown graphically in figure 1 where the indices of refraction $(N)$ have been plotted versus time and temperature. The equilibrium indices (line AC) for corresponding temperatures are shown on the left in figure 1 . The glass at temperature $T_{1}$, in equilibrium, will have a constant index of refraction A versus time. If the temperature is changed instantaneously to some lower value $T_{2}$, the index of refraction gradually increases to a new equilibrium value $C$ corresponding

${ }^{1}$ Figures in brackets indicate the literature references at the end of this paper.

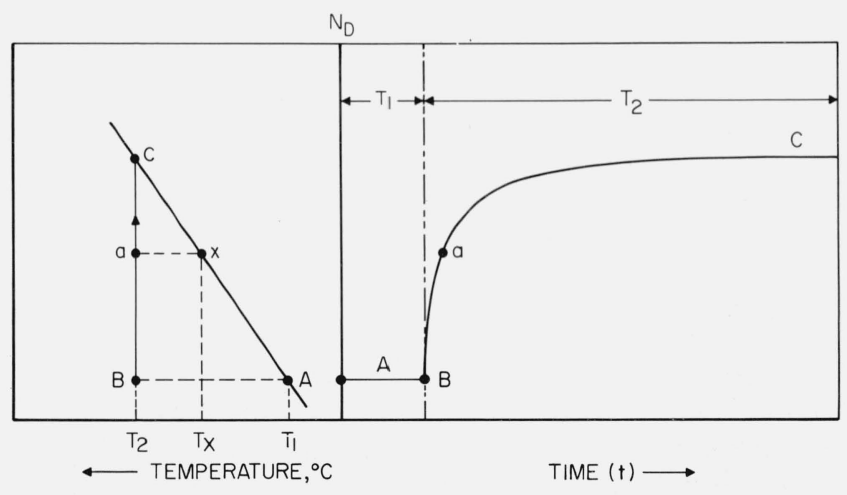

Figure 1. Plot of index of refraction versus temperature and time.

to the new temperature $T_{2}$. The time dependence $B-C$ of this property can be represented as either

(a) a linear (i.e., nonamplitude dependent) distribution of relaxation times or as

(b) a nonlinear single relaxation (i.e., at any instant there is only one relaxation time, but at subsequent intervals the relaxation time changes with the distance from equilibrium) [2].

Even though any curve can be represented by (a) or (b), a systematic series of such curves cannot be 
represented by either (a) or (b) [3] only. Unfortunately none of the previous investigators has been able to extract either the distribution of relaxation times or the nonlinear effects [3].

The purpose of this paper is to show how one can find a distribution of relaxation times from such annealing experiments.

The time dependence of the physical properties of liquids has also been investigated by measuring the frequency dependence of these same properties. The time and frequency experiments are equivalent and a mathematical procedure will be considered which can interrelate both types of measurements.

\section{Pertinent Viscoelastic Theory}

When a distribution of relaxation times is present in a fluid, the complex modulus of compression, $K^{*}$, is frequency dependent and can be written [4]

$$
\begin{array}{r}
K^{*}=K^{\prime}+i K^{\prime \prime}=K_{0}+\left(K_{\infty}-K_{0}\right)\left[\int_{0}^{\infty} \frac{g(\tau)(\omega \tau)^{2} d \tau}{1+(\omega \tau)^{2}}\right. \\
\left.+i \int_{0}^{\infty} \frac{g(\tau) \omega \tau d \tau}{1+(\omega \tau)^{2}}\right]
\end{array}
$$

where $K_{0}=\lim K^{*}$

$$
\omega \rightarrow 0 \text {, }
$$

$$
\begin{aligned}
K_{\infty}= & \lim K^{*} \\
& \omega \rightarrow \infty, \\
\omega= & \text { the angular frequency, } \\
\tau= & \text { the relaxation times, } \\
g(\tau)= & \text { the relaxation spectrum. }
\end{aligned}
$$

Although this is in many ways a convenient form in which to represent material properties, it is seldom possible to find a unique spectrum to represent a measured response function (either a steady-state function of frequency like the above or a transient function of time like the annealing measurements). This results both from the experimental uncertainty of the measured function and the fact that a complete response function is seldom measured. As an example of the difficulty of finding a unique spectrum, figure 2 due to Macedo and Litovitz [5] shows a plot of reduced modulus versus $\omega \bar{\tau}$, as well as two different $g(\tau)$. First, $g(\tau)$ was assumed to be of the form

$$
g(\tau)=(b /(\tau \sqrt{\pi})) \exp \left[-b^{2}\left(\ln \left(\tau / \tau^{\prime}\right)\right)^{2}\right]
$$

which is a symmetric spectrum in the logarithm of $\tau$ centered at $\tau^{\prime}$ whose width is determined by the value of $b$.

The second fit was obtained by using two delta functions for $g(\tau)$ such that

$$
K^{\prime}=K_{0}+\left(\frac{K_{\infty}-K_{0}}{2}\right)\left[\frac{\left(\omega \tau_{1}\right)^{2}}{1+\left(\omega \tau_{1}\right)^{2}}+\frac{\left(\omega \tau_{2}\right)^{2}}{1+\left(\omega \tau_{2}\right)^{2}}\right] .
$$

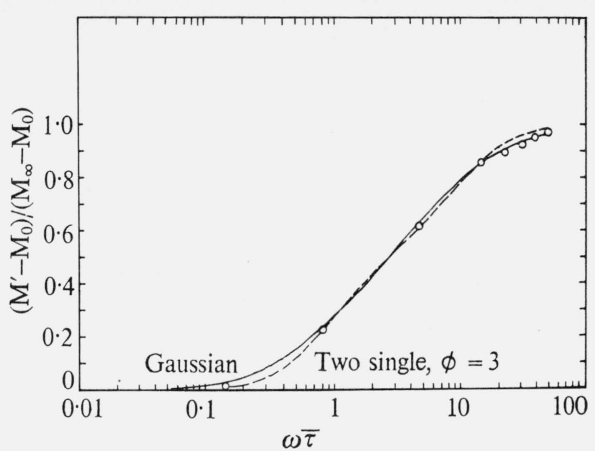

FiguRE 2. Normalized real part of the modulus versus normalized frequency for $\mathrm{B}_{2} 0_{3}$ at $550{ }^{\circ} \mathrm{C}$ from reference 5 . $\varphi=\left(\tau_{2} / \tau_{1}\right) .^{1 / 2}$

Depending on the experiment and subsequent data reduction, one can obtain the relaxation time spectrum at different thermal conditions. In the above example where the modulus was calculated from ultrasonic velocity measurements, one has a spectrum corresponding to a stress relaxation modulus at constant entropy and strain. In the annealing experiment one is measuring the relaxation of a thermally induced strain at constant temperature and stress. Even though there is a rigorous mathematical connection between the constant stress spectrum (retardation) and the constant strain spectrum (relaxation) at either constant temperature or constant entropy, no simple relation between the spectra for the two limiting thermal conditions is available.

\section{Analysis of Annealing Experiments}

\subsection{Thermal History of Crossover}

Spinner and Napolitano [1], following some work of Ritland [6] and Kovacs [7], transferred a sample from the middle of an approach curve having an index (a), see figure 1 , to a furnace whose temperature $\left(T_{x}\right)$ was so adjusted that the equilibrium value of the index $(x)$ was equal to the approach index $(a)$. This change in temperature was designated as a crossover. The changes in index with time at two of these crossovers are shown in figure 3 . The thermal history of these specimens follows:

(a) All specimens were held at $585^{\circ} \mathrm{C}$ until the structure was at equilibrium. (Index of refraction was constant with time.)

(b) The specimens were reintroduced into the furnace set at $470{ }^{\circ} \mathrm{C}$ for Crossover $\mathrm{A}$ and at $500{ }^{\circ} \mathrm{C}$ for Crossover B. The curves through the open circles represent the values of index of the specimens for the respective approach curves.

(c) The change in index with time was followed by frequent measurements. When the index had reached a value close to the equilibrium value for a temperature of $544{ }^{\circ} \mathrm{C}$, the sample was transferred to the furnace set at $544{ }^{\circ} \mathrm{C}$ and the subsequent change in index followed until it had reached its final equilibrium. 


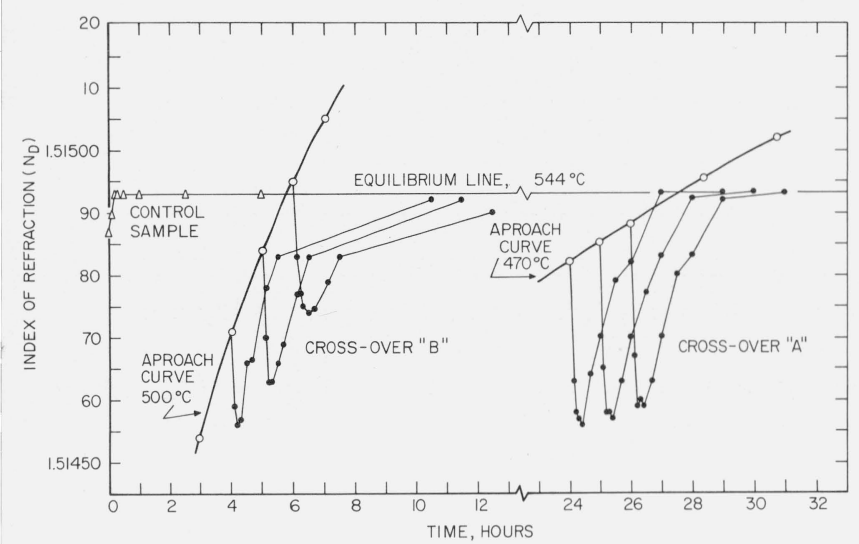

FigurE 3. Index versus time at two selected crossovers. Equilibrium temperature for both crossovers is $544{ }^{\circ} \mathrm{C}$. Approach temperature for crossover A is $470^{\circ} \mathrm{C}$, and for crossover $\mathrm{B}$, is $500^{\circ} \mathrm{C}$. Data from reference 1 .

Several different starting points for this final crossover curve (solid circles) were selected on each of the two approach curves, as shown in figure 3 .

\subsection{Effect of Distribution of $\tau$ on Annealing}

Reexamining figure 1, one observes that upon an instantaneous change in temperature from $T_{1}$ to $T_{2}$, a thermal strain of $N_{\infty}\left(T_{1}\right)-N_{\infty}\left(T_{2}\right),(B-C)$, is applied to the sample. We will proceed to study the decay or relaxation of this strain.

As noted in the introduction, one cannot represent a series of volume-time curves in terms of any given sum of exponentials with given relaxation times and weighting factors if the total volume change is very large. However, we might well expect, if the total range of volume covered by the final (crossover) curves is small, that a series of such curves could be represented by a sum of exponentials without changing the constants. We shall find that the curves analyzed here can be so represented for any given final equilibrium temperature, even though the various crossover curves are started from different points on the same approach curve or from points on two different approach curves. We will term such behavior "linear."

The simplest such representation which could describe the type of behavior shown in figure 3 is two exponential terms with equal weighting factors, which may be set equal to $1 / 2$. If we were describing the index-time relation between two equilibrium conditions (starting with the glass in equilibrium at one temperature and following the index as a function of time after suddenly changing the temperature to a second value which is then held constant), we would write:

$$
\begin{aligned}
N(t)=N_{\infty}+N\left(1 / 2 e^{-t / \tau_{1}}\right. & \left.+1 / 2 e^{-t / \tau_{2}}\right) \\
& =1 / 2\left(M_{1}(t)+M_{2}(t)\right)
\end{aligned}
$$

where $M_{i}(t)=N_{\infty}+N_{i} e^{-t / \tau_{i}}$

where $N_{1}=N_{2}=N$.
This formulation can be thought of as describing the relaxation (change of volume) to a final equilibrium value following a change in temperature (analogous to an isothermal recovery function) in terms of two separate mechanisms, the first of which relaxes appreciably faster than the second. $N_{1}$ and $N_{2}$ are measures of the total relaxation undergone by the two separate mechanisms in reaching equilibrium. If the starting point is one of equilibrium $\left(M_{1}(0)=M_{2}(0)\right)$ the two mechanisms will have to relax the same total amount, $N_{\infty}\left(T_{1}\right)-N_{\infty}\left(T_{2}\right)$, and $N_{1}$ will equal $N_{2}$. However, when starting from a nonequilibrium approach curve, $N_{1}$ and $N_{2}$ will depend on the particular approach curve being followed, as well as on the actual measured index at the crossover point, and in general, will not be equal. To represent this situation eq (4) can be generalized to:

$$
\begin{aligned}
N(t)=1 / 2\left(M_{1}(t)+M_{2}(t)\right) & =N_{\infty} \\
+ & 1 / 2\left(N_{1} e^{-t / \tau_{1}}+N_{2} e^{-t / \tau_{2}}\right) .
\end{aligned}
$$

Figure 4 shows schematically the time dependence of $N, M_{1}$, and $M_{2}$ for a glass initially at equilibrium. As the structure rearranges itself to the new equilibrium, $M_{1}$ will rise faster since it is associated with the shorter relaxation time $\tau_{1}$, while $M_{2}$ will change more slowly. Thus, around the bend of the approach curve, there will be the largest difference between $M_{1}$ and $M_{2}$ in the glass. As time progresses, $M_{1}$ will reach its equilibrium value for $T_{2}$ first and stay there. From then on the spread between $M_{1}$ and $M_{2}$ narrows because the slow relaxation process continuously approaches the same equilibrium value at a slower rate. Finally, when the structure is completely at the new equilibrium the spread becomes zero, and one has again an equilibrium glass $\left(M_{1}=M_{2}\right)$. At a crossover (a) the fast relaxation time $(\tau)$ corresponds to an index $M_{1}$ higher than the (average) measured value, $N$, and the slow relaxation time $\left(\tau_{2}\right)$ corresponds to an index $M_{2}$ lower than $N$.

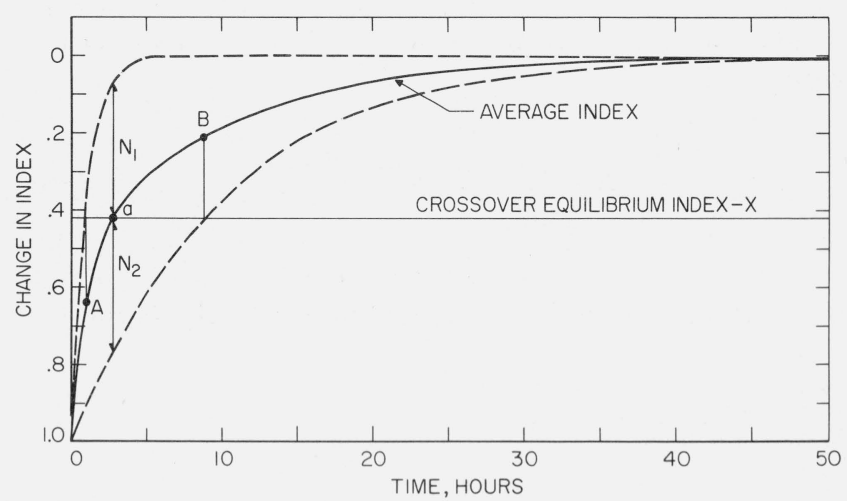

FIGURE 4. Illustration of two relaxation times represented by upper and lower dashed curves.

The average or measured index is given by the solid line. The crossover equilibrium index $x$ and the transfer point are also shown. 


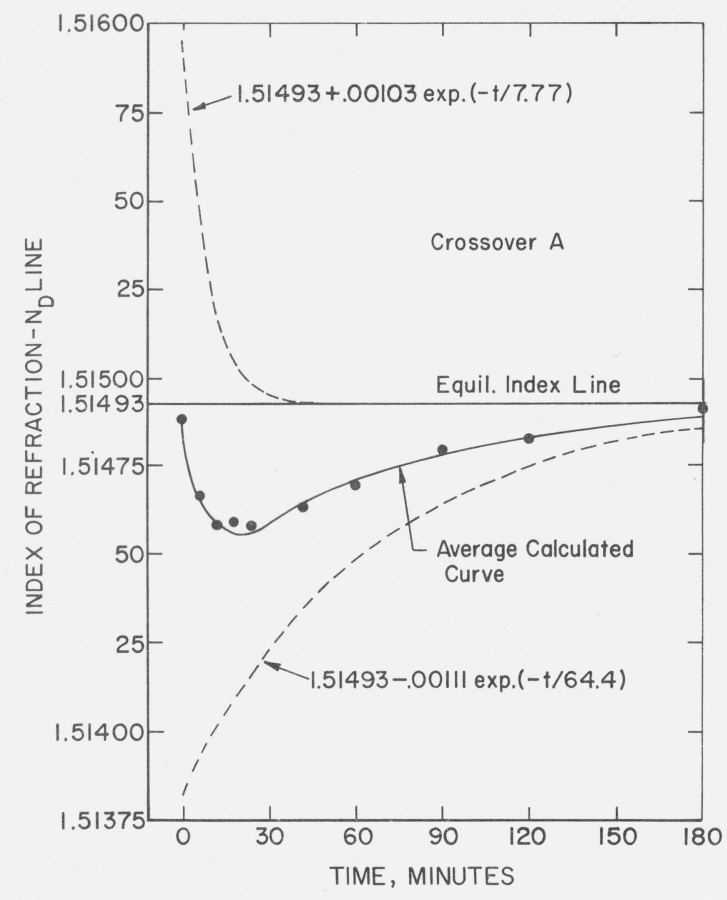

Figure 5. Crossover A-Solid curve is the average of the two dashed curves, each having a single relaxation time; the fast relaxation time $\left(\tau_{1}=7.77 \mathrm{~min}\right)$, above; and the slow relaxation time $\left(\tau_{2}=64.4\right.$ min) below the equilibrium line $\mathrm{N}_{x}=1.51493$.

Here $N_{1}=0.00103$ and $N_{2}=-0.00111$. Points are third experimental set from cross. over $\mathrm{A}$, in figure 4 . Data from reference 1 .

If the index at the point of crossover, $N(0)$, is equal to the equilibrium index, $N(\infty)$ at the crossover temperature $T_{x}$, then $N_{2}=-N_{1}$. A prerequisite for a minimum in the crossover is that $N_{1}$ and $N_{2}$ have opposite signs. This is fulfilled when $N$ has a value between $\mathrm{A}$ and $\mathrm{B}$ in figure 4.

The crossover curves were analyzed as follows:

(a) The logarithm of the difference between the equilibrium index and actual index was plotted as a function of time. From the best straight line through the final points, both $\tau_{2}$ and $N_{2}$ were calculated, assuming $t \gg \tau_{1}$ so $M_{1}(t)$ in eq (5) can be set equal to $N_{\infty}$.

(b) From the initial value of the index at crossover, $N_{0}$ and the equilibrium value, $N_{\infty}, N_{1}$ was calculated from

$$
\Delta=N_{0}-N_{\infty}=\frac{N_{1}+N_{2}}{2}
$$

(c) From the time, $t_{m}$, at which the index reached its minimum value, $\tau_{1}$ was calculated from the equation

$$
\begin{aligned}
& \frac{\partial N}{\partial t}=-\left[N_{1} \exp \left(-t_{m} / \tau_{1}\right)\right] / \tau_{1} \\
&-\left[N_{2} \exp \left(-t_{m} / \tau_{2}\right)\right] / \tau_{2}=0 .
\end{aligned}
$$

The results of this computation for the crossover A curve with $N_{0}$ closest to $N_{\infty}$ is shown in figure 5 . The solid line is the average of the upper curve, $M_{1}$, and the lower curve, $M_{2}$. The agreement of the solid calculated curve with the actual experimental points is seen to be good.

\subsection{Nonlinearity in Crossover Region}

The definition of linearity adopted requires that the relaxation times at a crossover are independent of amplitude (i.e., $N_{1}$ and $N_{2}$ ). Since in the linear case the relaxation times are characteristic of the equilibrium temperature rather than the approach curve, two crossovers having the same equilibrium temperature will have the same $\tau_{1}$ and $\tau_{2}$ and the same weighting factors $(1 / 2)$, regardless of approach temperature.

The two crossovers in figure 3 present themselves as a natural check for linearity. This is done by applying $\tau_{1}$ and $\tau_{2}$, already computed for crossover $\mathrm{A}$, to crossover B. Equations (5) and (6) were combined to give:

$$
\begin{aligned}
N-N_{\infty}=\frac{N_{1}}{2}\left[\exp \left(-t / \tau_{1}\right)-\right. & \left.\exp \left(-t / \tau_{2}\right)\right] \\
& +\Delta \exp \left(-t / \tau_{2}\right) .
\end{aligned}
$$

Solving for $N_{1}$ gives:

$$
N_{1}=2 \frac{\left(N-N_{\infty}\right)-\Delta \exp \left(-t / \tau_{2}\right)}{\exp \left(-t / \tau_{1}\right)-\exp \left(-t / \tau_{2}\right)}
$$

From this equation the average value of $N_{1}$ for crossover B was obtained using $\tau_{1}$ and $\tau_{2}$ from crossover A and $\Delta$ from eq 6 . Figure 6 compares the calculated curve (solid line) with the corresponding experimental points (the middle set from crossover B in fig. 3). This fit is seen to be comparable with that observed for crossover A (fig. 5). Linearity of the relaxation process in the crossover region with respect to a given equilibrium temperature is thus demonstrated to within the experimental uncertainty.

\subsection{Further Experiments}

Spinner and Napolitano (1) also reported data for a third crossover $(\mathrm{C})$ which corresponds to an initial equilibrium temperature of $585^{\circ} \mathrm{C}$, approach temperature $477^{\circ} \mathrm{C}$, and equilibrium $527^{\circ} \mathrm{C}$. The results of a calculation performed as above are shown in figure 7 . Inasmuch as this crossover contains the largest number of experimental points, the agreement of the computed curve with these experimental points is even more impressive than that for the other two crossovers shown. For this crossover, $\tau_{1}$ was computed to be 75 min and $\tau_{2}, 540 \mathrm{~min}$.

An encouraging result is that $\left(\tau_{2} / \tau_{1}\right)_{527{ }^{\circ} \mathrm{C}}=7.2$ is within the experimental uncertainty of the value of 8.3 found at $544{ }^{\circ} \mathrm{C}$. Since the distribution of relaxation times is temperature independent in most liquids, one can assume that this is the case here, even though the temperature range is not large enough to justify a conclusive statement. 


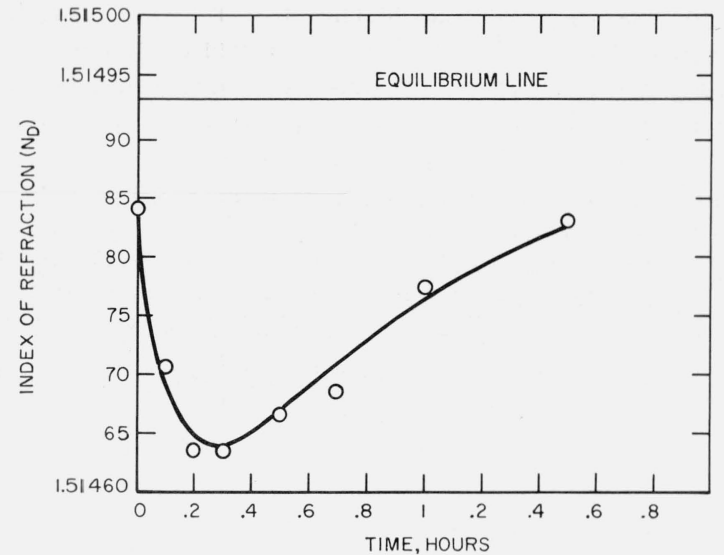

Figure 6. Crossover B-Solid curve is computed result using fast and slow relaxation times found for crossover $A$.

In this case $N_{1}=+0.00069$ and $N_{2}=-0.00088$. Points are middle experimental set (ref. [1]) from crossover B, in figure 4 .

The annealing experiments of Spinner and Napolitano are based on the following experiment of Ritland [6]. A sample of BSC glass was cooled through the glass transformation range at a constant rate. Later it was reintroduced in a furnace whose temperature matched the sample's fictive temperature. Figure 8 shows the time dependence of the specimen after reinsertion. Even though the dip is slightly shallower the curve is similar. Unfortunately, Ritland did not take enough data at "long" times for accurate description of $\tau_{2}$. Since the composition of Ritland's glass is very close to that of Spinner and Napolitano, it was decided to use the same ratio of $\tau_{2} / \tau_{1}=8$ and fit the dip by adjusting the average $\tau$ and the initial spread in index. Again, a good fit was obtained, indicating once more, that the distribution of relaxation times is a property of BSC glass and not of the thermal history.

\subsection{Comparison of $\bar{\tau}_{v s}$ and $\bar{\tau}_{s}$}

The distribution of volume relaxation times has been calculated at two temperatures $\left(544\right.$ and $\left.527{ }^{\circ} \mathrm{C}\right)$ for BSC glass. From these a weighted average relaxation time at constant pressure and temperature can be defined by:

$$
\bar{\tau}_{p T}=\Sigma g_{i} \tau_{i}=1 / 2 \tau_{1}+1 / 2 \tau_{2} .
$$

On the basis of the values of $\bar{\tau}_{p T}$ for each of these temperatures the apparent activation energy for volume relaxation, $H_{b}$, was computed from the well-known relation

$$
\bar{\tau}_{p T}=A e^{H b^{\prime R T}}
$$

where $A$ is an arbitrary constant. $H_{b}$ was found to be $155 \mathrm{kcal} / \mathrm{mole}\left(6.49 \times 10^{5} \mathrm{~J} / \mathrm{mole}\right)$. This value is comparable with the activation energy for shear viscosity, $H_{s}=140 \mathrm{kcal} / \mathrm{mole}\left(5.86 \times 10^{5} \mathrm{~J} / \mathrm{mole}\right)$, over about the same temperature range, based on direct measure-

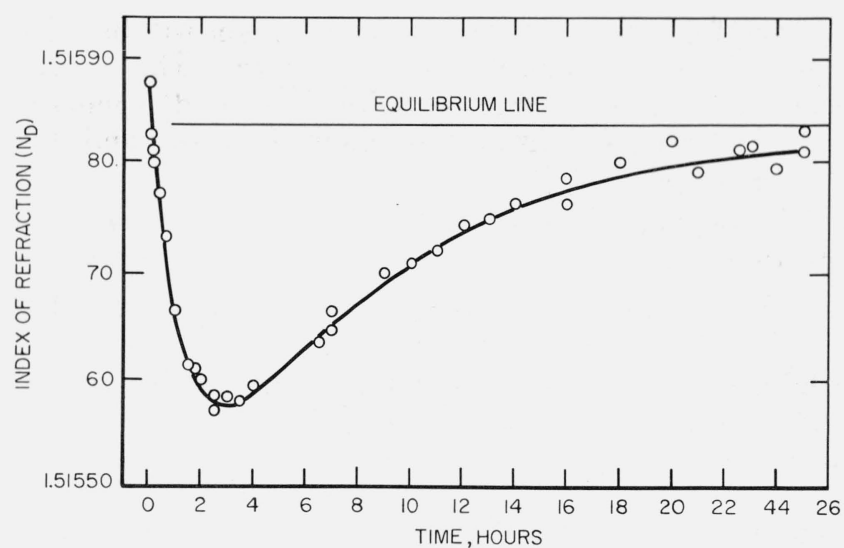

Figure 7. Crossover C-Equilibrium temperature $527{ }^{\circ} \mathrm{C}$ and $\mathrm{N}_{\infty}=1.51584$, approach temperature $477^{\circ} \mathrm{C}$.

Solid line is computed curve based on two single relaxation decay curves (not shown). and shows fit to experimental points (ref. [1]). Here $\tau_{1}=75 \min ; N_{1}=0.00094$; and $\tau_{2}=540 \mathrm{~min}, N_{2}=-0.00086$

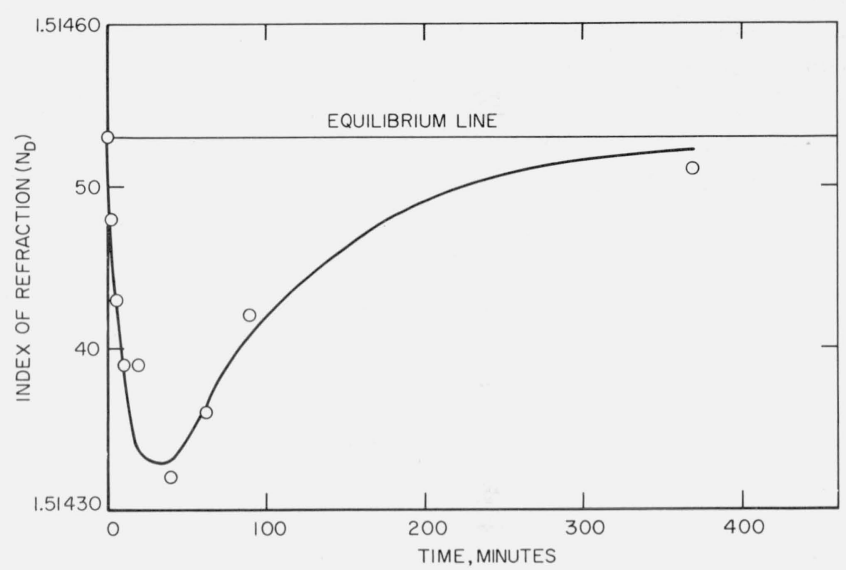

FIGURE 8. Solid line is computed curve based on two single relaxation decay curves.

Here $\tau_{1}=12 \mathrm{~min}, N_{1}=0.00064$ and $\tau_{2}=96 \mathrm{~min}, N_{2}=-0.00064, N_{\infty}=1.51453$, and shows fit to experimental points [6]. Initial distribution of index was obtained by rate cooling samples.

ment of the shear viscosity by the fiber elongation method [8].

According to a tabulation by Litovitz and Davis [9] the ratio of the average adiabatic isochoric relaxation time for dilatation, $\bar{\tau}_{v s}$, to Maxwell's average shear relaxation time, $\bar{\tau}_{s}$, turns out to be between about 0.79 and 4.60 for a number of associated liquids. Hence a calculation of this ratio should give us a check on whether the relaxation times as calculated here to represent thermally induced volume changes can reasonably be equated to relaxation times associated with purely mechanical deformations.

The isothermal isobaric average relaxation (retardation) time, $\bar{\tau}_{P T}$, at $544{ }^{\circ} \mathrm{C}$ was calculated from eq (10) to be $2.17 \times 10^{3} \mathrm{sec}$. It is assumed that the relationship

$$
\bar{\tau}_{P T} / \bar{\tau}_{v s}=\left(\kappa_{T_{0}} / \kappa_{T_{\infty}}\right)\left(C_{v_{0}} / C_{v_{\infty}}\right)
$$


derived for a single relaxation mechanism will be adequate for the comparison desired here. This gives, using isothermal compressibilities, $\kappa_{T}$, and isochoric specific heat, $C_{v}$, from references [11, 12, and 13] $\bar{\tau}_{v s}=653 \mathrm{sec}$.

The average shear relaxation time $\bar{\tau}_{s}$, was computed [4] from

$$
\bar{\tau}_{s}=\eta_{s} / G_{\infty}
$$

where $G_{\infty}$ is the glass modulus of rigidity [14] and at $544{ }^{\circ} \mathrm{C}$, was found to be $303 \mathrm{sec}$. Thus, the ratio $\bar{\tau}_{v s} / \bar{\tau}_{s}$, was 2.2 , a value in good agreement with the above mentioned Litovitz and Davis compilation for associated liquids. Therefore, the relaxation times calculated here can reasonably be equated with the relaxation process associated with purely mechanical deformations.

The width of the distribution found in BSC glass, as given by $\tau_{2} / \tau_{1}=8$ is comparable with that found in most liquids. For example, $\mathrm{B}_{2} \mathrm{O}_{3}$ at $550{ }^{\circ} \mathrm{C}$ (fig. 2) is a little wider, $\tau_{2} / \tau_{1}=9$, but at higher temperatures it is narrower [5]. The associated organic liquids, whose distributions are the best known, have about the same width provided they are monomeric [9]. For polymeric liquids this ratio is meaningless but would be from $10^{3}$ to $10^{6}$. Thus one can conclude that BSC is not polymeric.

\section{Discussion}

The only physical model that would readily predict two relaxation times would be two immiscible phases. Even though there is a possibility that these relaxation times be single they should be separated by many orders of magnitude, since one of the immiscible phase in the silicate system is usually almost pure $\mathrm{SiO}_{2}$ with its associated high viscosity (very long relaxation time). Also, had there been such an immiscibility then the temperature cycling of Spinner and Napolitano would not have been reproducible.

We will now present a microscopic model to explain the distribution of relaxation times. The topological model will consist of a distribution of micro regions in space, each with a different relaxation time. Such regions have been postulated by various authors. For example, Bockris et al. [15], pictured an "iceberg structure" for silicate glasses. Litovitz and McDuffie [16] and Macedo and Litovitz [5] have proposed that the distribution of relaxation times as well as the nonArrhenius temperature dependence of the structural relaxation time in associated liquids is due to the cooperative behavior of molecules and the possible existence of clusters.

In order to explain the conductivity experiments, which will be described below, the glass is assumed to have two microdisperse regions which are interconnected. This should be expected from both the immiscibility work of Haller [17] and the immiscibility precursor stage investigated by Ohlberg and Parsons [18]. The dimension of these micro regions must be smaller than $150 \AA$ in order not to be detected by light scattering or (ordinarily) by the electron microscope. (Zarzycki and Mezard [19] claim to have seen such regions even in single component glasses by an elaborate process using transmission in an electron microscope.) It is assumed that the relaxation time of each region is a function only of its own structure, i.e.,

$$
\tau_{i}=f\left(M_{i}, T\right) \neq f^{\prime}\left(M_{j \neq i}\right) .
$$

This equation is equivalent to assuming that the hybrid equation of Macedo and Litovitz [20] is good for each separate region, rather than for the average values.

Evidence for the topological model can be obtained from the conductivity experiments of Ritland [6]. These conductivity measurements were made well below the glass transition and there were no structural rearrangements taking place during the measurements. The conductivity of a glass below $T_{g}$ is known to be due to alkali diffusion through the immobile lattice. This ionic diffusion has been shown to have a distribution of relaxation times. From rate theory one can calculate the conductivity due to ionic jumps for a single relaxational process to be

$$
\sigma=\frac{a^{2} n}{c k T \tau_{I}}
$$

where $a$ is the "jump distance"; $n$, the number density of ions; $\tau_{I}$, the ionic relaxation time; and $c$, a geometrical constant. Let us consider the effect of these two microdispersed regions on the conductivity. First, whatever caused region one to have a shorter volume relaxation time, such as higher disorder, less intramolecular bonding, etc., may reasonably be assumed to cause the ionic relaxation time in region one to be shorter. Thus, one can write for the conductivity of each region:

$$
\sigma_{i}=\frac{a^{2} n}{c k T \tau_{I i}}
$$

where the nonindex quantities are the same for both regions. Since each region is interconnected, the measured conductivity can be approximated by

$$
\sigma=\sigma_{1}+\sigma_{2}=\frac{a^{2} n}{c k T} \quad\left(\frac{1}{\tau_{I_{1}}}+\frac{1}{\tau_{I_{2}}}\right) .
$$

In fact, if $\tau_{I_{1}} \cong(1 / 8) \tau_{I_{2}}$, then the conductivity is controlled primarily by $\tau_{I_{1}}$. Note that here is where the topological model differs from the phenomenological approach. Had one assumed a distribution of relaxation times in a homogeneous material then

$$
\sigma=\frac{a^{2} n}{c k T\left(\tau_{I_{1}}+\tau_{I_{2}}\right)}
$$

and, provided $8 \tau_{I_{1}} \cong \tau_{I_{2}}$, the conductivity would have been controlled by $\tau_{I_{2}}$. 
Figure 9 due to Ritland shows resistivity versus index for glasses that were either cooled at constant rate through the glass transformation region (rate cooled) or quenched from an equilibrium configuration (soaked). It can be seen from this figure that the variation of resistivity with thermal history for a given index is of the same order of magnitude as the variation with index for a given type of annealing experiment. The resistivity is not a single valued function of the average structure as represented by the index. Thus, any model in which the relaxation is a function of the average structure, such as Tool's fictive temperature [21], must be ruled out. In the topological model the conductivity is only a function of $\tau_{I_{1}}$ which in turn is a function of $M_{1}$ and not $M_{2}$.

In a previous section, it was found that the rate cooled glass with an index of 1.51453 had $M_{1}=1.51517$ associated with $\tau_{1}$ and $M_{2}=1.51389$ associated with $\tau_{2}$. Since as a first approximation resistivity is only controlled by structure 1 , the resistivity of the rate cooled sample having an index 1.51453 should be the same as that of the soaked sample having an index 1.51517 because both have the same values of $M_{1}$. This point is shown plotted in figure 9. If a line is drawn through the Ritland's soak specimen data points it will intercept his rate cooled curve. This intersection should only occur when the rate sample's cooling rate approaches the cooling rate of the chilled specimen. Therefore, these lines through the rate cooled and chilled specimen data points should be almost parallel. Thus for comparison a line is drawn parallel to the rate cooled specimen and through the "theoretical" point. Since this line is above one experimental point and below the other, it is considered within the experimental accuracy of the soaked data. Note, that eq (18) due to the phenomenological approach would predict that $\sigma$ is a function of either $M_{2}$ or $M_{1}+M_{2}$ depending on the assumed functional dependence of $\tau_{2}$, neither of which will fit the data. Even though the data are sparse in this experiment it has

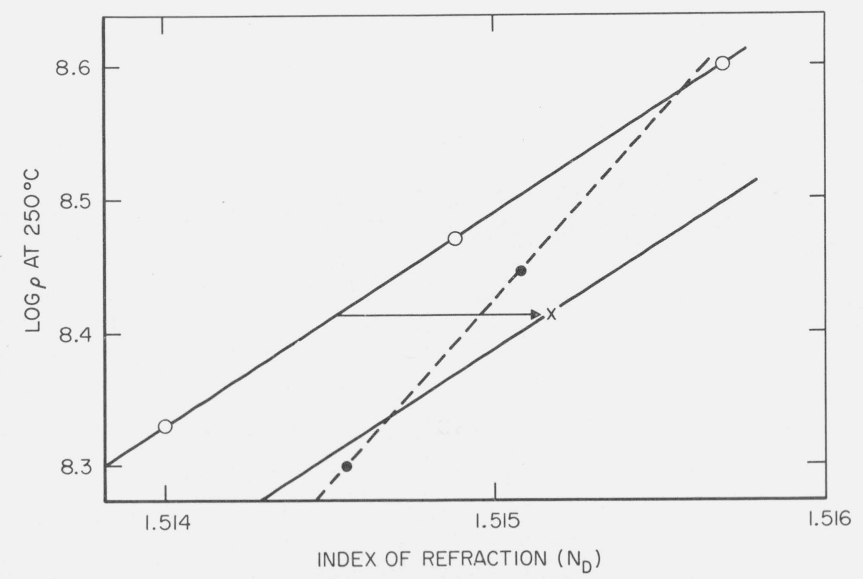

FIGURE 9. Resistivity versus refractive index for rate cooled, $\mathrm{O}$, and soak, samples; X predicted value. Data from reference $[6]$. been shown that the two-parameter topological model represents the state of the glass regardless of the heat treatment.

Rate Cooling. The best experiment to show whether the apparent temperature dependence of the viscosity is directly due to temperature or indirectly due to structure is the rate cooling process.

When a glass sample is cooled through the glass transition region at a constant rate, initially the sample will follow the equilibrium index curve (see fig. 10). When the long volume relaxation time, $\tau_{2}$, becomes comparable with the cooling rate, $M_{2}$ will lag behind the equilibrium value $N_{\infty}$. As the temperature continues to lower $\tau_{2}$ becomes longer and longer compared with the cooling rate, and eventually the structure is thermally arrested at a value of the relaxation time, $\tau_{R}$, which is governed by the cooling rate. Upon further cooling $M_{2}$ remains constant. $M_{1}$ will follow a similar history and $\tau_{1}$ will also be finally arrested at the same value, $\tau_{R}$.

Thus as a sample is cooled through the glass transition at a constant rate each relaxing process will be thermally arrested at the same value of relaxation time, $\tau_{R}$, which is a function of the cooling rate. If the values of the relaxation times are only a function of structure, then, at any temperature, all the relaxation times in a rate cooled glass cooled at the same rate should be equal. The crossover data of Ritland, figure 8, clearly demonstrates that this is not the case.

If we assume that the hybrid equation is completely controlled by the activation energy (i.e., $f(T)$ is given by eq (11)), the difference in index $M_{1}-M_{2}$ in the rate cooled glass represents the difference in temperatures required for $\tau_{1}$ to equal $\tau_{2}$. One, then, can write for the activation energy

$$
H_{b}=R \ln \left(\tau_{2} / \tau_{1}\right)\left\{1 / T_{1}-1 / T_{2}\right\}^{-1}
$$

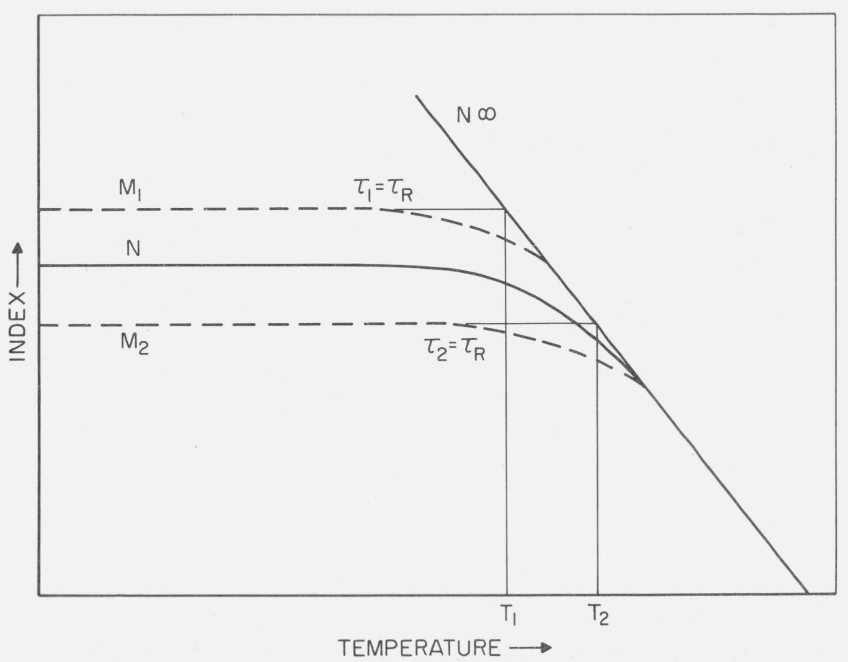

Figure 10. Plot of index versus temperature for a rate cooled sample.

Equilibrium index $\left(N_{\infty}\right)$ curve is also shown plotted. 
in which $T_{1}=T_{R}-N_{1} / \frac{\partial N}{\partial T}$ and similarly for $T_{2} ; T_{R}$ is the crossover temperature of Ritland; and $\partial N / \partial T$ was taken from Spinner and Napolitano. The activation energy calculated from eq (19) is $122 \mathrm{kcal} / \mathrm{mol}(5.12$ $\times 10^{5} \mathrm{~J} / \mathrm{mol}$ ) which corresponds favorably with the $140 \mathrm{kcal} / \mathrm{mol}\left(5.86 \times 10^{5} \mathrm{~J} / \mathrm{mol}\right)$ obtained from the viscosity data.

One must conclude that most of the apparent activation energy of viscous flow is due to a true activation energy effect. This is in direct contradiction with recent papers on the interpretation of the Fulcher equation by W.L.F. [22], Cohen and Turnbull [23], but agrees with the work of Maurer [24], who annealed glass under pressure.

\section{Conclusion}

It has been shown how one can obtain the distribution of relaxation times by proper analysis of annealing experiments. A physical model for the distribution was proposed, and it, the topological model, was found to explain not only the temperature dependence of viscosity, but also the resistivity experiments of Ritland. In doing so, Tool's fictive temperature was extended to two parameters, which can explain both equilibrium and nonequilibrium behavior of glasses. The temperature dependence of the viscosity was found to be due to a true activation energy rather than a free volume effect. The stress (thermal) strain experiment gives a distribution many decades narrower than the stress (pressure) strain work previously done $[25,26]$ in the relaxation region. Since no pressure was applied, no "delayed elastic relaxation" takes place in the thermal experiment. Thus, there is the added advantage of only measuring the structural distribution of relaxation times.

This analysis was applied to a borosilicate crown glass, and it was found that $\tau_{2} / \tau_{1} \approx 8$ (Gaussian $b=0.6$ ). This narrow distribution indicates that the flow in this material is monomeric rather than polymeric $\left(10^{3}<\tau_{2} / \tau_{1} \leqq 10^{6}\right)$. The monomeric nature of silicate glasses has been postulated by Bockris et al. [15], from zero frequency compressibility measurements at high temperatures, but was questioned when Westman [27] proved that phosphate glasses are polymeric. The present work together with
Macedo and Litovitz [5] distribution of relaxation times in $\mathrm{B}_{2} \mathrm{O}_{3}$ indicates that the iceberg picture of Bockris is correct for the borates and silicates. The ratio of the average volume to average shear relaxation time was found to be $\approx 2$, in $\operatorname{good}$ agreement with other associated liquids [9].

\section{References}

[1] S. Spinner and A. Napolitano, J. Res. NBS 70A (Phys. and Chem.), No. 2, 147-152 (1966)

[2] E. U. Condon, The Glass Industry 33, 307, 322-3 (1952).

[3] M. Goldstein, Modern Aspects of the Vitreous State, Vol. 3, pp. 90-125 (Butterworths, Washington, D.C., 1964)

[4] K. F. Herzfeld and T. A. Litovitz, Absorption and Dispersion of Ultrasonic Waves (Academic Press, New York and London, 1959).

[5] P. B. Macedo and T. A. Litovitz, Phys. and Chem. of Glasses 6, 69 (1965)

[6] H. N. Ritland, J. Am. Ceram. Soc. 39, 403 (1956).

[7] A. J. Kovacs, Fortschr. Hockpolym-Forsch. 3, 394 (1963).

[8] A. Napolitano and E. G. Hawkins, J. Res. NBS 68A (Phys. and Chem.), No. 5, 439-448(1964).

[9] T. A. Litovitz and C. M. Davis, Physical Acoustics, Vol. II, ch. 5 (Academic Press, New York and London, 1965).

[10] H. J. Bauer, Physical Acoustics, Vol. II, ch. 2 (Academic Press, New York and London, 1965).

[11] Handbook of Glass Manufacture, F. V. Tooley, editor (Ogden Publishing Co., New York, N.Y., 1953).

[12] A. Cooper, private communication.

[13] H. N. Ritland, J. Am. Ceram. Soc. 37, 370 (1954)

[14] S. Spinner, J. Am. Ceram. Soc. 39, 113 (1956).

[15] J. O'M Bockris and Kojoneu, J. Phys. Chem. 82, 4493 (1960).

[16] T. A. Litovitz and G. McDuffie, J. Chem. Phys. 39, 729 (1963).

[17] W. Haller, J. Chem. Phys. 42, 686 (1965).

[18] S. M. Ohlberg and J. M. Parsons, Physics of Non-Crystalline Solids, Delf Conference (North Holland Publishing Co., Amsterdam, 1964).

[19] J. Zarzycki and R. Mezard, J. Phys. and Chem. of Glasses 3, 163 (1962).

[20] P. B. Macedo and T. A. Litovitz, J. Chem. Phys. 42, 245 (1965).

[21] (a) A. Q. Tool, J. Am. Ceram. Soc. 29, 240 (1948). (b) A. Q. Tool, L. W. Tilton and J. B. Saunders, J. Res. NBS 38, 519 (1947) RP1793.

[22] M. L. Williams, R. F. Landel, and J. D. Ferry, J. Am. Chem. Soc. 77, 3701 (1955).

[23] M. H. Cohen and D. Turnbull, J. Chem. Phys. 31, 1164 (1959).

[24] R. D. Maurer, J. Am. Ceram. Soc. 40, 211 (1957).

[25] C. R. Kurkjian, Phys. and Chem. of Glasses 4, 128 (1963).

[26] J. DeBast and P. Gilard, Phys. and Chem. of Glasses 4, 117 (1963).

[27] A. E. R. Westman, Modern Aspects of the Vitreous State, Vol. I, pp 63-91 (Butterworths, W ashington, D.C. 1960).

(Paper 71A3-456) 